\title{
Modeling the Internet
}

\author{
Pamela Zave \\ AT\&T Laboratories-Research \\ www2.research.att.com/ pamela
}

\begin{abstract}
The Internet has changed the world. Its astounding success has led to explosive growth in users, traffic, and applications, which has made its original architecture and protocols obsolete. Currently the networking community is questioning all aspects of Internet technology, as researchers and stakeholders try to understand how to meet new requirements for functionality, quality of service, availability, and security.

In this technological crisis, one of our most powerful technical tools, namely functional modeling (as opposed to performance modeling), is being completely ignored. In this talk I explain how modeling can be put to good use in the Internet context, how the culture of the Internet Engineering Task Force and the networking research community resist such efforts, and what might be done to bring about a cultural change.

The talk will be illustrated with examples and results from several projects using different modeling languages and techniques. These include:

- A project to understand, formalize, and partially verify SIP, the dominant protocol for IP-based multimedia applications. The official specification of SIP consists of many thousands of pages of English text.

- A project revealing many unknown defects in the Chord routing protocol, which is the most-cited protocol for maintenance of peerto-peer networks.

- A project to generate modular telecommunication services from models in a high-level, domain-specific language. This long-term project, based on the DFC architecture, has already produced two large-scale deployed systems.

- A project to discover how to meet complex requirements for application sessions using software composition.

The descriptions of these projects will emphasize topics such as the search for the right modeling language and the search for principles of networking.
\end{abstract}

